\title{
Inhaled but not intravenous milrinone prevents pulmonary endothelial dysfunction after cardiopulmonary bypass
}

\author{
Y. Lamarche, MD, ${ }^{a, b}$ O. Malo, MSc, ${ }^{a}$ E. Thorin, $\mathrm{PhD}^{, b}$ A. Denault, MD, ${ }^{d}$ M. Carrier, MD, MSc, ${ }^{a, b}$ J. Roy, CPP,
} and L. P. Perrault, MD, PhD ${ }^{a, b, c}$

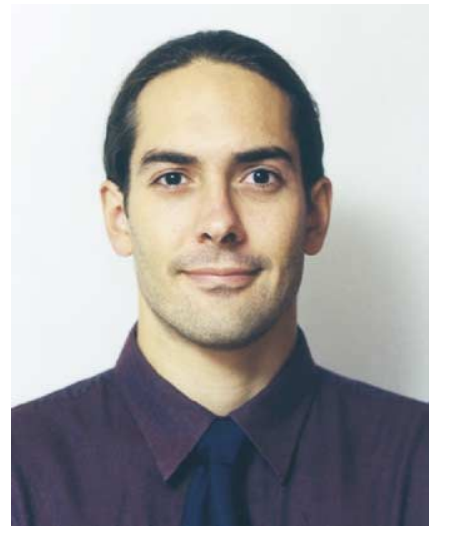

Dr Lamarche
From the Research Center ${ }^{\mathrm{a}}$ and the Department of Surgery, ${ }^{\mathrm{b}}$ Montreal Heart Institute; the Department of Pharmacology, ${ }^{\mathrm{c}}$ University of Montreal; and the Departments of Anesthesiology ${ }^{\mathrm{d}}$ and Perfusion, ${ }^{\mathrm{e}}$ Montreal Heart Institute, Montreal, Quebec, Canada.

Received for publication Feb 3, 2004; revisions received Aug 25, 2004; accepted for publication Sept 2, 2004.

Address for reprints: Dr Louis P. Perrault, Research Center, Montreal Heart Institute, 5000 Belanger St East, Montreal, Quebec, H1T 1C8, Canada (E-mail: louis. perrault@icm-mhi.org).

J Thorac Cardiovasc Surg 2005;130:83-92

$0022-5223 / \$ 30.00$

Copyright (C) 2005 by The American Association for Thoracic Surgery

doi:10.1016/j.jtcvs.2004.09.011
Objective: Cardiopulmonary bypass triggers a systemic inflammatory response that alters pulmonary endothelial function, which can contribute to pulmonary hypertension. Milrinone is a type III phosphodiesterase inhibitor. The objective of this study was to compare the effects of inhaled and intravenous milrinone on the pulmonary endothelium-dependent relaxations and hemodynamic and oxygenation parameters after cardiopulmonary bypass in a porcine model.

Methods: Five groups of Landrace swine were compared: (1) control group, no cardiopulmonary bypass; (2) bypass group, 90 minutes of normothermic bypass and 60 minutes of reperfusion; (3) inhaled milrinone group, bypass preceded by a 1.8-mg bolus of inhaled milrinone followed by a continuous milrinone nebulization; (4) intravenous milrinone group, bypass preceded by $2 \mathrm{mg}$ of intravenous milrinone; and (5) inhaled $\mathrm{NaCl}$ group, bypass preceded by inhaled saline solution. After sacrifice, pulmonary arterial endothelium-dependent relaxations to acetylcholine and bradykinin were studied in organ chambers.

Results: Inhaled milrinone caused less hypotension $(P<.05)$, a lesser decrease in peripheral vascular resistances $(P<.01)$, and a lower heart rate $(P<.05)$ than intravenous milrinone. Inhaled milrinone prevented the alterations in relaxations of pulmonary arteries to acetylcholine caused by cardiopulmonary bypass, and relaxations to bradykinin were improved in the inhaled milrinone group $(P<.05)$ compared with the cardiopulmonary bypass and control groups.

Conclusions: Inhaled milrinone prevents the occurrence of the pulmonary endothelial dysfunction seen after cardiopulmonary bypass. The hemodynamic and oxygenation profiles obtained with inhaled milrinone are safer than with intravenous milrinone. These strategies might be useful in preventing pulmonary hypertension after cardiac surgery.

$\mathrm{C}$ ardiopulmonary bypass $(\mathrm{CPB})$ induces a systemic inflammatory response that affects all organ systems. The physiologic alterations after CPB were recognized early after the development of CPB in the 1950s. The postpump syndrome is characterized by an increase in pulmonary capillary permeability, leading to decreased oxygenation, increased alveolar-arterial oxygen gradient, decreased pulmonary compliance, and an increased pulmonary vascular resistance. ${ }^{1,2}$ Among the most important repercussions of the inflammatory cascade are those on the pulmonary vasculature. During CPB, blood flow is diverted from the right atrium to the CPB pump, goes through an oxygenator membrane, and is pumped back into the aorta, and thus the lungs are minimally perfused during CPB. At separation from CPB, the lungs are reperfused and experience ischemia-reperfusion injury caused by exposure to large amounts of free radicals. Because of the contact of the blood elements with the nonphysiologic surface of the bypass circuit, neutrophils and platelets are activated and contribute to pulmonary damage, which triggers endothelial dysfunction after $\mathrm{CPB} .{ }^{3,4}$ After $\mathrm{CPB}$, the damage to 
TABLE 1. Hemodynamic data throughout the experiment

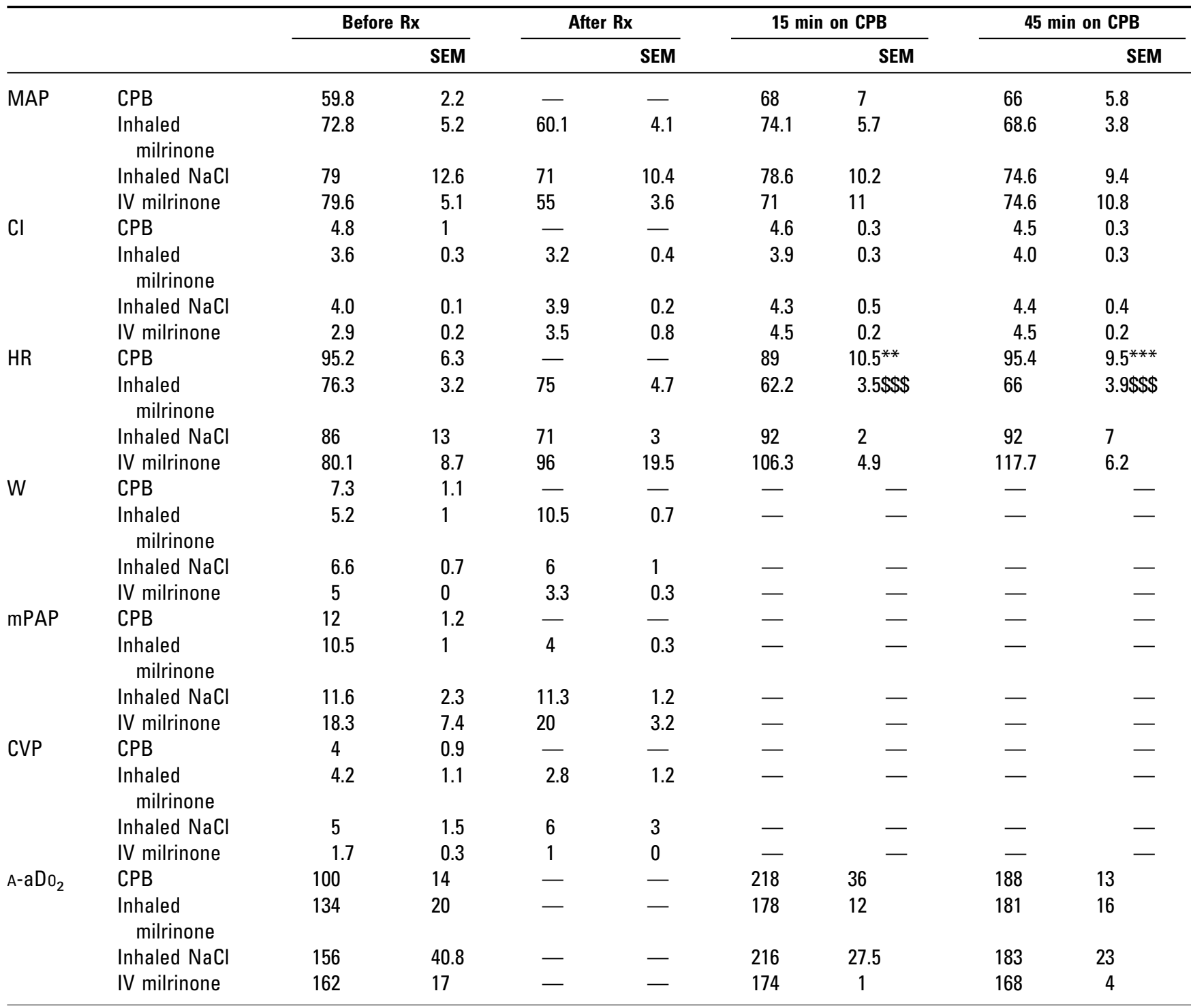

$R x$, Experimental drug; $C P B$, mean cardiopulmonary bypass; $M A P$, mean arterial pressure; $I V$, intravenous; $C l$, cardiac index; $H R$, heart rate; $W$, wedge pressure; $m P A P$, pulmonary artery pressure; $C V P$, central venous pressure; $A-a D 0_{2}$, alveolar-arterial oxygen gradient. $* P<.05$ versus inhaled milrinone. $* * P<.01$ versus inhaled milrinone. $* * * P<.001$ versus inhaled milrinone. $\$ P<.05$ versus intravenous milrinone. $\$ \$ \$ P<.001$ versus intravenous milrinone. $\& \& P<.01$ versus CPB. $\$ P<.05$ versus $\mathrm{NaCl} . \$ \$ P<.011$ versus $\mathrm{NaCl} . \$ \$ \$ P<.001$ versus $\mathrm{NaCl}$.

the pulmonary endothelium can lead to pulmonary hypertension $(\mathrm{PH})$, leading to an increase in right ventricular work. Right ventricular dysfunction after CPB carries a poor prognosis, with a perioperative mortality ranging from $44 \%$ to $86 \%$. $^{5}$

The endothelium has an important role in regulating the vascular tone by releasing several vasorelaxing substances, such as nitric oxide (NO) and prostacyclin $\left(\mathrm{PGI}_{2}\right)$, and vasoconstrictors, such as endothelin and thromboxanes. The ability to induce vasorelaxation can be decreased when endothelial dysfunction is present. ${ }^{6}$ Several pharmacologic agents have been used to limit the increase in pulmonary vascular resistance and $\mathrm{PH}$ after cardiac surgery, including intravenous nitroglycerin, intravenous milrinone, inhaled $\mathrm{NO}$, and inhaled $\mathrm{PGI}_{2}$.

Milrinone, a phosphodiesterase III inhibitor that increases intracellular levels of cyclic adenosine monophosphate (cAMP), induces positive cardiac inotropy and systemic vasorelaxation. When administered intravenously, milrinone decreases systemic vascular resistances, causing hypotension, which can be hazardous in the hours after cardiac surgery. The use of inhaled milrinone has recently been described by Haraldsson and colleagues ${ }^{7}$ to avoid the systemic hypotension associated with intravenous milrinone. They observed a de- 


\begin{tabular}{|c|c|c|c|c|c|}
\hline \multicolumn{2}{|c|}{$75 \min$ on CPB } & \multicolumn{2}{|c|}{$30 \mathrm{~min}$ after CPB } & \multicolumn{2}{|c|}{60 min after CPB } \\
\hline & SEM & & SEM & & SEM \\
\hline 70.4 & 5.3 & 85.6 & 5.1 & 61.4 & $4.4^{*} \$$ \\
\hline 68 & 3.2 & 65.33 & 6.1 & 54.8 & 3.5 \\
\hline 84.6 & 6.2 & 73.5 & 5.3 & 62 & 5.5 \\
\hline 75 & 8.6 & 61 & 14.8 & 44.6 & 13.7 \\
\hline 4.85 & 0.3 & 7.2 & 0.7 & 4.8 & $0.2^{* *} \$ \$$ \\
\hline 4.04 & 0.2 & 3.9 & 0.4 & 4.4 & 0.3 \\
\hline 4.6 & 0.3 & 6.21 & 1.7 & 3.9 & 0.3 \\
\hline 4.6 & 0.2 & 3.89 & 0.6 & 3.3 & 1.3 \\
\hline 112.4 & $4.9^{* * *} \$$ & 92.2 & $5.9^{*}$ & 92.6 & $2.5^{*}$ \\
\hline 64.7 & $3.8 \$ \$ \$$ & 73.7 & $3.4 \$ \$ \$$ & 73.7 & $4.6 \$$ \\
\hline 98 & 12 & 115 & 24 & 106 & 13 \\
\hline 141.3 & 14.3 & 108.7 & 10.3 & 95.3 & 4.6 \\
\hline - & - & 5.5 & 2.1 & 3.9 & 1.4 \\
\hline- & - & 2.5 & 0.7 & 2.4 & 0.6 \\
\hline - & - & 7.5 & 2 & 3.5 & 1.22 \\
\hline - & - & 4 & 1.6 & 11.7 & 0.4 \\
\hline - & - & 17.6 & 0.8 & 14 & 1.4 \\
\hline- & - & 13 & 0.9 & 13.1 & 1.07 \\
\hline - & - & 13.7 & 2 & 13.7 & 2 \\
\hline - & - & 15.7 & 1.2 & 17.3 & 1.9 \\
\hline - & - & 4.7 & 1.5 & 3 & 0.5 \\
\hline- & - & 2.3 & 0.7 & 2 & 0.8 \\
\hline - & - & 3.5 & 2.9 & 2.6 & 1.2 \\
\hline - & - & 5.7 & 4.3 & 5.7 & 3.7 \\
\hline 196 & 17 & 181 & 31 & 156 & 23 \\
\hline 186 & 20 & 165 & 17 & 193 & 27 \\
\hline 193.7 & 22.7 & 216.2 & 6.8 & 199.8 & 12.2 \\
\hline 160 & 7 & 277 & 92 & 360 & $56 \& \& * * \$$ \\
\hline
\end{tabular}

crease in pulmonary artery pressure (PAP) without systemic hypotension. In a recent randomized controlled trial comparing the use of intravenous milrinone with that of placebo in patients with congestive heart failure, intravenous milrinone lowered the systemic arterial pressure and accelerated the heart rate, but there was no clinical benefit compared with placebo. ${ }^{8}$ Our main hypothesis is that inhaled milrinone could prevent post-CPB pulmonary endothelial dysfunction and reduce the pulmonary arterial pressure without the systemic side effects known for intravenous milrinone in patients undergoing cardiac surgery. The aim of the present study was to compare the effects of inhaled and intravenous milrinone in a swine model of CPB on pulmonary endothelial function, hemodynamics, and oxygenation. The levels of cAMP were also compared to document the mechanism of action of the drug.

\section{Material and Methods}

Experimental Preparation for All Groups (Anesthesia) All experiments were performed with Landrace swine (McGill University, Montreal, Quebec, Canada) of either sex aged 8 weeks and weighing $25 \pm 3 \mathrm{~kg}$. Animals were maintained and tested in accordance with the recommendations of the guidelines on the 
TABLE 2. Contractile responses to $\mathrm{KCl}$ and PE

\begin{tabular}{lllll}
\hline & Control & CPB & \multicolumn{1}{c}{$\begin{array}{c}\text { Inhaled } \\
\text { milrinone }\end{array}$} & $\begin{array}{c}\text { Intravenous } \\
\text { milrinone }\end{array}$ \\
\hline $\mathrm{KCl}(\mathrm{g})$ & $5.1 \pm 0.3$ & $3.9 \pm 2.2$ & $4.8 \pm 0.28$ & $4.0 \pm 0.3$ \\
$\mathrm{PE}(\mathrm{g})$ & $3.0 \pm 0.2$ & $2.5 \pm 0.1$ & $2.7 \pm 0.1$ & $2.4 \pm 0.1$ \\
Dose PE $\left(\times 10^{-7} \mathrm{~mol} / \mathrm{L}\right)$ & $5.5 \pm 0.9$ & $7.0 \pm 9.6$ & $8.3 \pm 0.93^{*, * *}$ & $4.1 \pm 0.3$ \\
\hline
\end{tabular}

Contractile responses to a depolarizing physiologic solution containing $60 \mathrm{mmol} / \mathrm{L} \mathrm{KCl}$ and concentration of phenylephrine required to achieve $50 \%$ of $\mathrm{KCl}$-induced contraction of pulmonary arteries isolated from control pigs $(\mathrm{n}=6)$ and pigs that underwent cardiopulmonary bypass ( $\mathrm{n}=6)$ with or without previous inhaled $(\mathrm{n}=3)$ or intravenous $(\mathrm{n}=3)$ administration of milrinone. $K C l$, Potassium chloride; $P E$, phenylephrine; $C P B$, cardiopulmonary bypass. Data are expressed as means $\pm \mathrm{SD}: * P<.01$ versus intravenous milrinone; $* * P<.05$ versus control.

Care and Use of Laboratory Animals issued by the Canadian Council on Animals and approved by a local ethics committee. The piglets were fasted for 12 hours before the operation and were sedated with intramuscular ketamine hydrochloride $(25 \mathrm{mg} / \mathrm{kg}$; Ayerst Veterinary Laboratories, Guelph, Ontario, Canada) and xylazine $(10 \mathrm{mg} / \mathrm{kg}$; Boehringer Ingelheim, Burlington, Ontario, Canada). Induction was achieved by using mask ventilation with 2\% isoflurane (Abbott Laboratories Limited, St-Laurent, Quebec, Canada).

The animals were subsequently intubated and mechanically ventilated with a constant oxygen and air mixture $3: 2$ or fraction of inspired oxygen $=0.66$ ) at 14 breath strokes per minute and a tidal volume of 6 to $8 \mathrm{~mL} / \mathrm{kg}$. Anesthesia was maintained with $1 \%$ isoflurane inhalation. Skin was prepared and draped with a sterile field. Arterial and venous blood gases were measured at regular intervals and maintained within physiologic limits by adjusting ventilation rate and tidal volume. An electrocardiogram was recorded from 4 subcutaneous limb and 1 precordial electrodes.

\section{Experimental Groups}

Group 1: Control $(\boldsymbol{n}=\mathbf{6})$. After skin preparation, the mediastinum was exposed through a median sternotomy. Heparin (300 IU/kg; Leo Pharma, Inc, Ajax, Ontario, Canada) was administered intravenously. After 1 hour of general anesthesia with $1 \%$ isoflurane, the animals were exsanguinated, and the lungs were harvested.

Group 2: CPB (90 minutes, 60 minutes of reperfusion; $\boldsymbol{n}=\boldsymbol{6}$ ). After skin preparation and draping with sterile fields, the jugular vein and carotid artery were cannulated to obtain a central venous line and arterial pressure, respectively. A cystostomy was performed for a measurement of urine output. A median sternotomy was performed, and the pericardium was opened for heart exposure.

A Swan-Ganz catheter (Edwards Lifesciences, Irving, Calif) was inserted through the jugular vein to measure PAP. After heparin administration (400 IU/kg), a double purse string was made on the proximal ascending aorta, and a single purse string was made on the right atrium. A blood sample was drawn thereafter from the right atrium, and proper anticoagulation was assessed with an activated coagulation time (ACT) by using the Hemochron 801 (Technidyne, Dorval, Quebec, Canada). The aorta and right atrium were cannulated when the ACT was greater than 300 seconds with 22F and 29/29F double-staged cannulas (DLP, Inc, Grand Rapids, Mich), respectively. After cannulation, CPB was initiated when the ACT was greater than 400 seconds. Ventilation was stopped throughout the CPB period. Anesthesia was maintained with the jugular vein line, with a continuous infusion of propofol (0.1-0.2 $\mathrm{mg} \cdot \mathrm{kg}^{-1} \cdot \mathrm{min}^{-1}$; Pharmascience, Inc, Montreal, Quebec, Canada). The CPB circuit consisted of a hollow-fiber membrane oxygenator with an incorporated filtered hardshell venous reservoir (Monolyth; Sorin, Irvine, Calif), a heatercooler, and a roller pump (Sarns 7000, Ann Harbor, Mich). The circuit was primed with $500 \mathrm{~mL}$ of Pentaspan (10\% Pentastarch; DuPont Pharma, Inc, Mississauga, Ontario, Canada), $250 \mathrm{~mL}$ of lactated Ringer's solution, $5000 \mathrm{IU}$ of heparin, $12.5 \mathrm{~g}$ of mannitol, and $15 / \mathrm{mEq}$ of sodium bicarbonate. After initial stabilization, the pump flow was adjusted to maintain an index of $2.4 \mathrm{~L} \cdot \mathrm{min}^{-1}$. $\mathrm{m}^{-2}$ and assessed on the basis of venous gases to maintain a mixed

\footnotetext{
Figure 1. A, Rings of porcine pulmonary with endothelium. $X$-axis, Log acetylcholine (mol/L); $Y$-axis, tension (percent contraction to phenylephrine); $P E$, phenylephrine. Data are expressed as means $\pm S E M$. ${ }^{*} P<.05, C P B$ versus inhaled milrinone; ${ }^{*} \boldsymbol{P}<.01, \mathrm{CPB}$ versus inhaled milrinone; ${ }^{* *} \boldsymbol{P}<.001$, CPB versus inhaled milrinone; $+\boldsymbol{P}$ $<.05$, inhaled versus intravenous milrinone; $++P<.01$, inhaled versus intravenous milrinone; $\Uparrow P<.05$, control

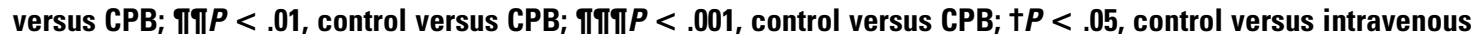
milrinone; $¥ P<.05$, control versus intravenous milrinone. $B$, Rings of porcine pulmonary with endothelium. $X$-axis, Log bradykinin (mol/L); $Y$-axis, tension (percent contraction to phenylephrine); $P E$, phenylephrine. Data are expressed as means \pm SEM. ${ }^{*} P<.05$, CPB versus inhaled milrinone; ${ }^{*} P<.01$, CPB versus inhaled milrinone; $+P$ $<.05$, inhaled versus intravenous milrinone; $++P<.01$, inhaled versus intravenous milrinone; $+++P<.001$, inhaled versus intravenous milrinone; \#P $<.05$, CPB versus intravenous milrinone; \#P $<.01$, CPB versus intravenous milrinone; $\Pi P<.05$, control versus $\mathrm{CPB} ; / P<.05$, control versus inhaled milrinone; $/ / P<.01$, control versus inhaled milrinone; $I / I P<.001$, control versus inhaled milrinone; $+P<.05$, control versus intravenous milrinone; $¥ P<.01$, control versus intravenous milrinone; $t+t P<.001$, control versus intravenous milrinone.
} 


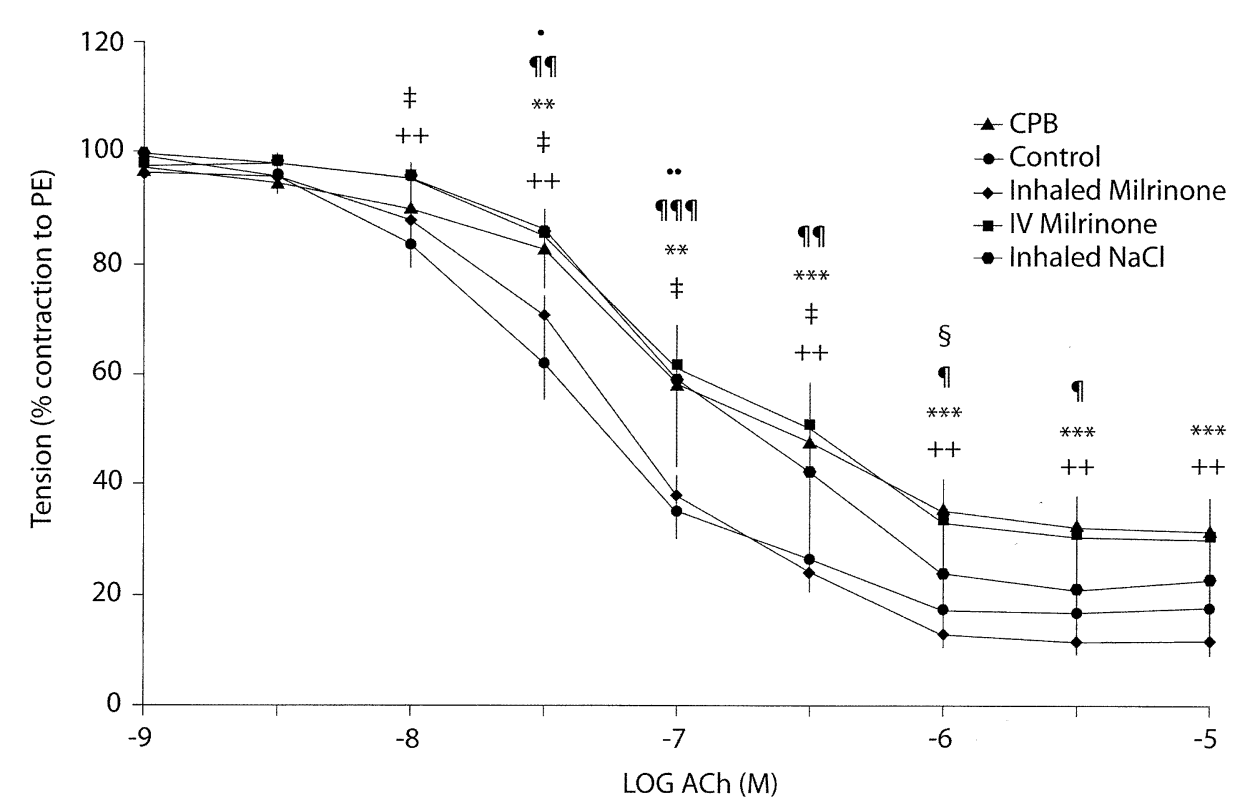

* $\quad p<0.05$ CPB vs Inhaled Milrinone

** $p<0.01$ CPB vs Inhaled Milrinone

*** $p<0.001$ CPB vs Inhaled Milrinone

$+\quad p<0.05$ Inhaled vs IV Milrinone

A ++ $p<0.01$ Inhaled vs IV Milrinone
I $\mathrm{p}<0.05$ Control vs CPB

กी $p<0.01$ Control vs CPB

กๆๆ $p<0.001$ Control vs CPB

$+\quad p<0.05$ Control vs IV Milrinone

$\neq \quad p<0.01$ Control vs IV Milrinone
- $\quad \mathrm{p}<0.01$ Control vs $\mathrm{NaCl}$

.. $\quad p<0.05$ Control vs $\mathrm{NaCl}$

$\S \quad \mathrm{p}<0.05$ Inhaled Milrinone vs $\mathrm{NaCl}$

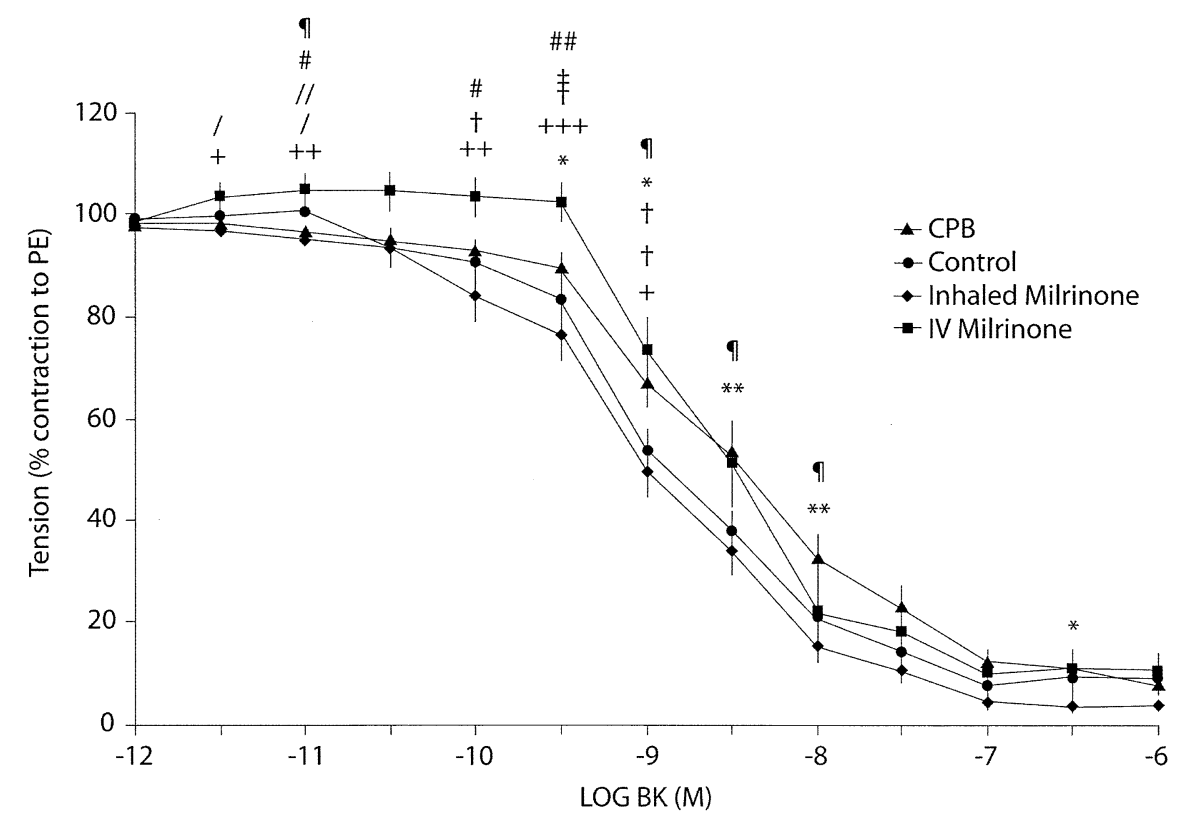

* $\quad p<0.05$ CPB vs Inhaled Milrinone

** $\quad p<0.01$ CPB vs Inhaled Milrinone

$+\quad p<0.05$ Inhaled vs IV Milrinone

$++\quad p<0.01$ Inhaled vs IV Milrinone

$+++p<0.001$ Inhaled vs IV Milrinone

\# $\quad \mathrm{p}<0.05$ CPB vs IV Milrinone

B \#\# $p<0.01$ CPB vs IV Milrinone ${ }^{\dagger}$

I $\mathrm{p}<0.05$ Control vs CPB

/ $\mathrm{p}<0.05$ Control vs Inhaled Milrinone

// $\mathrm{p}<0.01$ Control vs Inhaled Milrinone

/// $p<0.001$ Control vs Inhaled Milrinone

$+p<0.05$ Control vs IV Milrinone

$\neq \quad p<0.01$ Control vs IV Milrinone $p<0.001$ Control vs IV Milrinone

Figure 1. For legend see opposite page. 


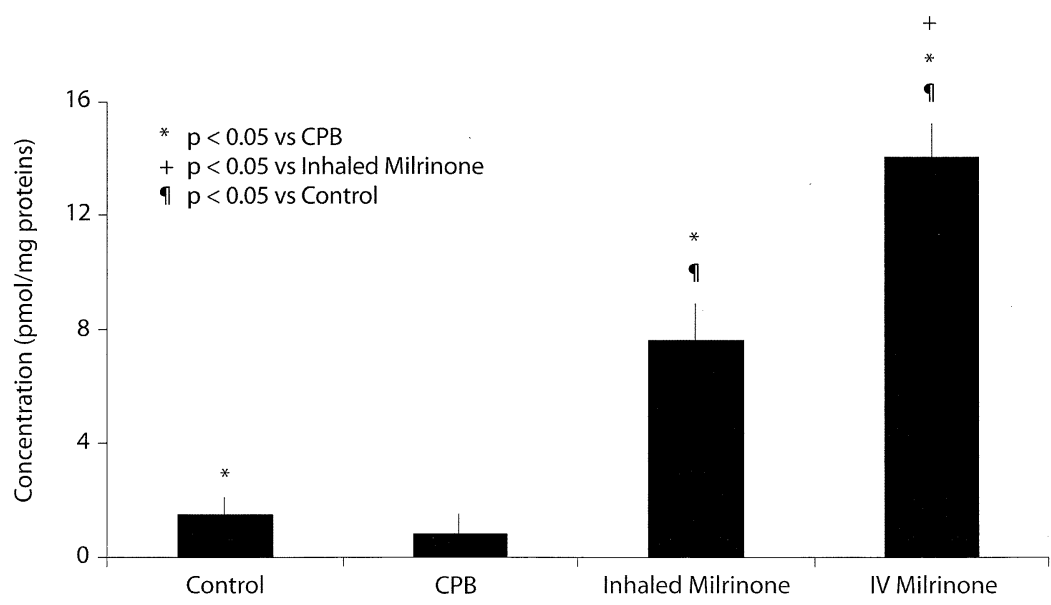

Figure 2. Concentration of cAMP in pulmonary arteries after CPB. $X$-axis, Study groups; $Y$-axis, cAMP concentration (pmol/mg proteins). Data are expressed as means \pm SEM. ${ }^{*} P<.05$ versus $\mathrm{CPB} ;+\boldsymbol{P}<.05$ versus inhaled milrinone; $\Uparrow \boldsymbol{P}<.05$ versus control.

venous saturation of greater than $60 \%$. Mean systemic arterial pressure was maintained between 50 and $70 \mathrm{~mm} \mathrm{Hg}$ with Ringer's lactate and punctual boluses of 50 to $200 \mu \mathrm{g}$ of neosynephrine (Cayman Chemical Co, Ann Arbor, Mich). The temperature was allowed to drift to $36^{\circ} \mathrm{C}$. The heart was left beating and empty. No aortic crossclamping or cardioplegia was used. Before CPB weaning, swine were rewarmed to $38^{\circ} \mathrm{C}$ (normal porcine temperature). After 90 minutes of $\mathrm{CPB}$, mechanical ventilation and isofluraneinduced anesthesia were reinstituted, and the animals were weaned from CPB. Normal circulation was restored for 60 minutes, at which time the animal was exsanguinated into the cardiotomy reservoir. The heart and lungs were excised en bloc and immediately immersed in a cold modified Krebs-bicarbonate solution ( $\mathrm{NaCl}, 118.3 \mathrm{mmol} / \mathrm{L} ; \mathrm{KCl}, 4.7 \mathrm{mmol} / \mathrm{L} ; \mathrm{MgSO}_{4}, 1.2 \mathrm{mmol} / \mathrm{L}$; $\mathrm{KH}_{2} \mathrm{PO}_{4}, 1.2 \mathrm{mmol} / \mathrm{L}$; glucose, $11.1 \mathrm{mmol} / \mathrm{L} ; \mathrm{CaCl}_{2}, 2.5 \mathrm{mmol} / \mathrm{L}$; $\mathrm{NaHCO}_{3}, 25 \mathrm{mmol} / \mathrm{L}$; and ethylenediamine tetraacetic acid, 0.026 $\mathrm{mmol} / \mathrm{L})$.

Group 3: $C P B$ and inhaled milrinone $(n=6)$. The same procedure was followed as in the CPB group (group 2), with the addition of a bolus of $1.8 \mathrm{mg}(60-90 \mu \mathrm{g} / \mathrm{kg})$ of milrinone (Primacor; Sanofi-Synthelabo Canada, Inc, Markham, Ontario, Canada) that was administered through the endotracheal tube through a nebulizer during the 15 minutes preceding the initiation of CPB. Milrinone was given as a dilution of $2 \mathrm{mg}$ of milrinone, $1 \mathrm{mg} / \mathrm{mL}$, diluted in $8 \mathrm{~mL}$ of normal saline $(200$ $\mu \mathrm{g} / \mathrm{mL}$ ). The drug was administered through a conventional in-line nebulizer (Salter Labs) connected to the inspiratory limb of the ventilator. Ventilation was stopped, and a continuous nebulization of milrinone at a rate of 0.08 to $0.11 \mu \mathrm{g} \cdot \mathrm{kg}^{-1}$. $\min ^{-1}$ with a continuous positive airway pressure of $3 \mathrm{~cm} \mathrm{H}_{2} \mathrm{O}$ was then instituted until the end of the $\mathrm{CPB}$. Before weaning from $\mathrm{CPB}$, nebulization was stopped, and ventilation was reinstituted.

Group 4: CPB with intravenous milrinone $(\boldsymbol{n}=3)$. The same procedure was followed as in the CPB group (group 2) with the addition of a $2-\mathrm{mg}(67-100 \mu \mathrm{g} / \mathrm{kg})$ bolus of milrinone diluted in 10
$\mathrm{mL}$ of saline solution and administered intravenously over 15 minutes after the administration of heparin and before the institution of CPB.

Group 5: CPB with inhaled vehicle $(0.9 \% \mathrm{NaCl}, \mathrm{n}=3)$. The same procedure was followed as in the CPB group (group 2) with the addition of a bolus of $8 \mathrm{~mL}$ of normal saline $(0.9 \% \mathrm{NaCl})$ as the vehicle used for administration of inhaled milrinone administered through the endotracheal tube through a nebulizer during the 15 minutes preceding the initiation of CPB. The saline was administered through a conventional inline nebulizer (Salter Labs) connected to the inspiratory limb of the ventilator. Ventilation was stopped, and a continuous nebulization of saline at a rate of 1.3 $\mathrm{mL} / \mathrm{h}$ with a continuous positive airway pressure of $3 \mathrm{~cm} \mathrm{H}_{2} \mathrm{O}$ was then instituted until the end of CPB. Before weaning from $\mathrm{CPB}$, nebulization was stopped, and ventilation was reinstituted.

\section{Hemodynamic and Biochemical Data}

The heart rate was continuously monitored from 5 subcutaneous limb electrodes. Arterial and venous blood gases were measured at regular intervals during the experiment (baseline; during CPB at 15,45 , and 75 minutes; and at 30 and 60 minutes after weaning from $\mathrm{CPB}$ ) and maintained within physiologic limits by adjusting the ventilation rate and tidal volume. Hemodynamic parameters, including mean arterial pressure, heart rate, mean PAP, central venous pressure, and pulmonary artery wedge pressure, were measured with a Swan-Ganz catheter at different time intervals during the procedure: after induction, after drug administration, and after weaning of CPB (30 and 60 minutes).

\section{Vascular Reactivity Studies}

Less than 10 minutes after en bloc excision, the heart was removed, and the primary pulmonary artery was dissected. Branches of second-degree pulmonary arteries were isolated and dissected free of connective and adventitial tissue and divided into rings ( $4 \mathrm{~mm}$ wide, 16 rings per animal). All rings 
were placed in organ chambers (Emka Technologies, Inc, Paris, France) filled with $20 \mathrm{~mL}$ of modified Krebs-bicarbonate solution continuously heated at $37^{\circ} \mathrm{C}$ and oxygenated with a carbogen mixture $\left(95 \% \mathrm{O}_{2}\right.$ and $\left.5 \% \mathrm{CO}_{2}\right)$. The rings were suspended between 2 metal stirrups, with the upper one connected to an isometric force transducer connected to a signal amplifier and then allowed to stabilize for 30 minutes. Data were collected with biologic signal data acquisition software (IOX 1.700; Emka Technologies, Inc). Each arterial ring was stretched to the optimal point of its active length-tension curve $(3.5 \mathrm{~g})$, as determined by measuring the contraction to $60 \mathrm{mmol} / \mathrm{L} \mathrm{KCl}$ at different levels of stretch. The maximal contraction of rings was then obtained with the addition of $\mathrm{KCl}(60 \mathrm{mmol} / \mathrm{L} \mathrm{KCl})$. After obtaining a plateau, all baths were washed twice with modified Krebs-bicarbonate solution, and indomethacin (INN: indometacin; $10^{-5} \mathrm{mmol} / \mathrm{L}$ ) to exclude production of endogenous prostanoids was added in each bath. After 45 minutes of stabilization, phenylephrine (range, $2 \times 10^{-7} \mathrm{~mol} / \mathrm{L}$ to $3 \times 10^{-6}$ $\mathrm{mol} / \mathrm{L}$ ) was added to obtain a contraction averaging $50 \%$ of the maximal contraction to $\mathrm{KCl}$.

\section{Endothelium-dependent Relaxations}

The NO-mediated relaxation pathway was studied by constructing concentration-response curves to acetylcholine $\left(10^{-9}\right.$ to $10^{-5}$ $\mathrm{mol} / \mathrm{L}$; an agonist of $\mathrm{M}_{2}$ receptors coupled to $\mathrm{Gi}$ proteins) and bradykinin $\left(10^{-12}\right.$ to $10^{-6} \mathrm{~mol} / \mathrm{L}$; an agonist of $\mathrm{B}_{2}$ receptors coupled to $\mathrm{Gq}$ proteins).

\section{Endothelium-independent Relaxations}

At the end of the experiment, endothelium-independent relaxations were studied with the use of $10^{-5} \mathrm{~mol} / \mathrm{L}$ sodium nitroprusside (SNP), an NO donor.

\section{Study Drugs}

All drugs were prepared daily. Acetylcholine, bradykinin, indomethacin, and SNP were obtained from Sigma Chemical Co (Oakville, Ontario, Canada). Propranolol was obtained from Biomol Research Laboratories, Inc (Plymouth Meeting, Pa), and phenylephrine was obtained from Cayman Chemical Co. Milrinone was obtained from Sanofi-Synthelabo.

\section{Determination of Pulmonary Artery Intravascular cAMP Content}

Rings from the 4 groups were collected after death, frozen in liquid nitrogen, and stored at $-70^{\circ} \mathrm{C}$ to determine the vascular cAMP content of porcine pulmonary arteries. At the time of analysis, all segments were pulverized in a liquid nitrogen-cooled stainlesssteel mortar and then transferred in trichloracetic acid solution $(6.25 \% \mathrm{wt} / \mathrm{vol})$. The acid extracts were then centrifuged at $4^{\circ} \mathrm{C}$ for 15 minutes at $12,000 \mathrm{~g}(3000 \mathrm{rpm})$ to precipitate cell debris and proteins. The pellets were used for total protein determination by using the Bradford microassay technique (Bio-Rad, Mississauga, Ontario, Canada). The supernatants were extracted 4 times with water-saturated diethyl ether to remove trichloracetic acid. Any residual diethyl ether was removed by heating the samples to $90^{\circ} \mathrm{C}$ for 3 to 5 minutes. cAMP quantitation was done by using an enzyme immunoassay system with acetylation based on rabbit anti-cAMP antibodies (Amersham Pharmacia Biotech, Baie
d'Urfé, Quebec, Canada). The amount of cAMP in each blood vessel ring was standardized to picomoles of cAMP per milligram of protein.

\section{Statistical Analysis}

All values are expressed as means \pm SEM. Contractions to phenylephrine are expressed as a percentage of the maximal contraction to $\mathrm{KCl}(60 \mathrm{mmol} / \mathrm{L})$. Relaxations are expressed as the percentage of the maximal contraction to phenylephrine for each ring. Two-way repeated analysis of variance was performed to compare each point of the concentration-response curves between control rings and CPB rings. The Student $t$ test for paired-unpaired observations was used for the comparison of the PAPs and the intravascular cAMP content. Statistical analysis was performed with the computer software SAS (SAS Instruments, Inc, Cary, NC).

\section{Results}

All the hemodynamic data are presented in Table 1 .

\section{Hemodynamic and Biochemical Data}

Inhaled and intravenous milrinone reduced mean arterial pressure. There was a more important decrease $(P<.05)$ in the intravenous milrinone group $(55 \pm 6 \mathrm{~mm} \mathrm{Hg})$ compared with that seen in the inhaled group $(60 \pm 10 \mathrm{~mm} \mathrm{Hg})$ from a preadministration mean arterial pressure of $75 \pm 10 \mathrm{~mm}$ $\mathrm{Hg}$. A decrease in systemic vascular resistance from $38 \pm 6$ to $23 \pm 5$ dynes $\cdot \mathrm{s}^{-1} \mathrm{~cm}^{-5} \cdot \mathrm{m}^{2}$ in the intravenous milrinone group was observed, whereas the systemic vascular resistance did not change in the inhaled milrinone group $(P<.01)$.

\section{Hemodynamic Data During and After Bypass}

Blood pressure and cardiac index. In the CPB group the mean arterial pressure and cardiac index were stable throughout the study period, except for 30 minutes after weaning of $\mathrm{CPB}$, when there was an increase in systemic blood pressure $(P<.05)$ and cardiac index $(P<.01)$. Inhaled and intravenous milrinone blunted the peak observed 30 minutes after the end of bypass.

Heart rate. There was a nonsignificant $(P=.13)$ increase in the heart rate during the bypass run in the CPB group. The heart rate was increased $(P<.05)$ in the intravenous milrinone group compared with in the CPB group and reached statistical significance only after 75 minutes of bypass $(P<.05)$. The heart rate was significantly lower in the inhaled milrinone group compared with in the CPB and intravenous milrinone groups at 15 , 45 , and 75 minutes during $\mathrm{CPB}$ and at 30 and 60 minutes after CPB $(P<0.05)$.

Oxygen exchanges. The alveolar-arterial oxygen gradient was significantly greater in the intravenous milrinone group 60 minutes after bypass $(P<.05)$ compared with in the CPB and inhaled milrinone groups. Oxygen 
exchanges were not different in the inhaled milrinone and CPB groups.

There was no statistical difference in PAP, pulmonary artery occlusion pressure (wedge), and central venous pressure during the experiment within or between the groups (data not shown).

\section{Vascular Reactivity Studies}

Contractions. The amplitude of contraction induced by $\mathrm{KCl}(60 \mathrm{mmol} / \mathrm{L})$ was similar in all groups (Table 2$)$. The concentration of PE necessary to reach $50 \%$ of the contraction induced by $60 \mathrm{mmol} / \mathrm{L} \mathrm{KCl}$ was greater in the inhaled milrinone group compared with in the control group $(P<.05)$ and the intravenous milrinone group $(P$ $<.01)$.

Relaxation. CPB induced an endothelial dysfunction, as suggested by the reduced potency and efficacy of acetylcholine $(P<.05$; Figure $1, A)$. This dysfunction was prevented by inhaled milrinone $(P<.05)$ but not intravenous milrinone.

Bradykinin-induced relaxations were also altered by CPB (Fig 1, B). Inhaled milrinone increased relaxation to bradykinin compared with that seen in all other groups $(P<.05)$. In addition, no statistically significant differences in relaxation to SNP were observed between groups, with all rings achieving 100\% relaxation (data not shown).

cAMP measurements. The cAMP content was significantly lower in the CPB group than in the control group ( $P$ $<.05)$. The cAMP content in the intravenous milrinone group was similar to that seen in the control group. The inhaled milrinone group had a higher content of cAMP than the CPB group but less than the $\mathrm{CPB}$ and the intravenous milrinone groups $(P<.05$, Figure 2$)$.

\section{Discussion}

The aim of this study was to compare the effects of preoperative inhaled and intravenous milrinone on the endothelial dysfunction of pulmonary arteries induced by $\mathrm{CPB}$. The major findings of this study are that (1) CPB induces a pulmonary endothelial dysfunction of the acetylcholine (an agonist of $\mathrm{M}_{2}$ receptor coupled to $\mathrm{Gi}$ proteins) and bradykinin (an agonist of $\mathrm{B}_{2}$ receptors coupled to $\mathrm{Gq}$ protein) pathways that is reversed by administration of inhaled but not intravenous milrinone before CPB; (2) inhaled milrinone is associated with better hemodynamic and oxygenation profiles than intravenous milrinone, with less hypotension, a lower heart rate, and a lesser reduction in systemic vascular resistances; and (3) intravenous milrinone causes an increase in the alveolar-arterial oxygen gradient.

These observations suggest that the administration of inhaled milrinone before CPB prevents pulmonary endothe- lial dysfunction with a superior pharmacodynamic profile than the administration of intravenous milrinone.

Cardiac surgery with CPB can be associated with the occurrence of PH. PH increases right ventricular work, which can lead to right ventricular dysfunction, which carries a poor prognosis and a perioperative mortality ranging from $44 \%$ to $86 \% .{ }^{6,9}$ Morita and colleagues ${ }^{10}$ have demonstrated in a porcine model that CPB causes a significant increase in pulmonary vascular resistance and decreases the right ventricular function by more than $50 \%$. Pulmonary artery endothelial dysfunction caused by a decrease in the production or increased degradation of relaxing factors results in $\mathrm{PH}$, leading to right ventricular dysfunction and low cardiac output syndrome. ${ }^{11}$ Consequently, prevention of $\mathrm{PH}$ after $\mathrm{CPB}$ is of utmost clinical importance, particularly in patients with preexisting $\mathrm{PH}$.

Two modes of administration of the frequently used drug milrinone were compared in a model with CPB. The dose used was similar to human doses, approximately 0.5 $\mu \mathrm{g} / \mathrm{kg}$ for the loading dose. To date, only one study had reported the use of inhaled milrinone after cardiac surgery. ${ }^{7}$ Thus far, no experimental study had been performed in a relevant animal model comparing 2 modes of administration of milrinone in a reproducible swine model of CPB associated with postoperative pulmonary endothelial dysfunction.

In this study the doses of phenylephrine used to contract the pulmonary arteries were higher in the inhaled milrinone group than in the control and intravenous milrinone groups. These higher doses might reflect a relative pulmonary vasoplegia caused by an increased bioavailability of cAMP (Figure 2). A lesser response of vascular smooth muscle cells to contracting agents could have beneficial implications in states of lower endothelial NO production, as observed with the lower pulmonary vascular resistance in the inhaled milrinone group. The reductions in pulmonary resistance and right-sided afterload observed in the inhaled milrinone group are the likely causes of the observed increased stroke volume, which is a favorable event in the postoperative setting.

The impairment of acetylcholine- and bradykinininduced endothelium-dependent relaxations in the CPB group compared with in the control group was prevented by inhaled milrinone but not by intravenous milrinone. Milrinone increases cAMP levels in smooth muscle cells, leading to vasorelaxation. The present experiments were performed in the absence of $\mathrm{PGI}_{2}$ production because of the use of indomethacin. Consequently, the increased relaxations are most likely caused by increased NO production or an augmented smooth muscle response to NO. Fortier and colleagues ${ }^{12}$ recently showed that the use of inhaled $\mathrm{PGI}_{2}$ before CPB increased endothelium-dependent relaxations to 
bradykinin through increasing cAMP levels. Niwano and associates ${ }^{13}$ described a cAMP-responsive element in the endothelial NO synthase promoter by using beraprost, a $\mathrm{PGI}_{2}$ receptor agonist, to enhance cAMP levels, which increased exhaled nitric oxide synthase expression and enhanced NO bioavailability. Hence by augmenting cAMP levels, milrinone might promote endothelial NO production, whereas selective administration in the pulmonary tree limits the systemic side effects.

The speculative reasons why the 2 modes of administration of milrinone achieved different effects on endothelium-dependent relaxations are numerous. Because intravenous milrinone has an important distribution volume, the precise amount of this drug reaching the lungs is unknown. Furthermore, tachycardia and hypotension could adversely affect the pulmonary endothelial function. Inhaled milrinone was administered as a bolus before initiation of CPB and followed by a continuous nebulization throughout $\mathrm{CPB}$. The dosages were extrapolated from human dosages and previous local experiments. The amount of milrinone reaching the lungs by means of nebulization was not quantified in the present experiments. Administration of milrinone through nebulization should not induce V/Q mismatch because only vessels of ventilated regions of the lung are reached by the molecule. On the other hand, intravenous milrinone caused general vasodilatation, resulting in shunting and a higher alveolar-arterial oxygen gradient. We believe that the initial bolus of inhaled milrinone is responsible for the majority of the effect compared with the continuous nebulization because the $3 \mathrm{~cm} \mathrm{H}_{2} \mathrm{O}$ positive end-expiratory pressure applied during CPB does not favor penetration of the particles in the parenchyma. Furthermore, only $10 \%$ of the initial dose of milrinone was nebulized over the total CPB time, corresponding to approximately $15 \%$ of the pharmacologic dose of intravenous milrinone.

Haraldsson and colleagues ${ }^{7}$ reported a reduction in pulmonary vascular resistance without side effects in patients with $\mathrm{PH}$ receiving inhaled milrinone. No changes in mean PAP were observed in this healthy swine model, as well as no side effects. Beneficial effects in terms of oxygenation and heart rate were observed. The wide variation between the animals' mean PAPs and the absence of pre-existing PH could partially explain the lack of significant change in mean PAP.

The smaller reduction in arterial pressure and in systemic vascular resistance in the inhaled milrinone group compared with intravenous administration is interesting because intravenous milrinone causes a significant systemic vasodilatation. Inhaled milrinone is associated with a safer profile, with lesser systemic actions.

The tachycardia associated with $\mathrm{CPB}$ was prevented by inhaled milrinone, whereas intravenous milrinone increased the heart rate compared with that seen in the $\mathrm{CPB}$ alone group. The decrease in myocardial oxygen demand associated with a slower heart rate is an additional advantage of inhaled milrinone.

\section{Clinical Relevance}

CPB is used every day in cardiac surgery. Despite a relatively low prevalence of postoperative $\mathrm{PH}$, various degrees of pulmonary endothelial dysfunction occur in some subsets or all of the patients with or without clinically apparent manifestations. On the other hand, risk factors for postoperative $\mathrm{PH}$ are well known, and the patients could benefit from prophylactic agents to lower their risk of development of this important complication and its deleterious consequences on right ventricular function. This article presents a new mode of administration for milrinone, a drug used for years in cardiac surgery. Inhaled milrinone is more effective and associated with less hypotension than the traditional intravenous route. In addition, inhaled milrinone is not associated with the alterations in oxygen exchange seen with intravenous administration and reverses the pulmonary endothelial dysfunction through prevention of ischemiareperfusion injury during $\mathrm{CPB}$.

\section{Limitations}

The pharmacokinetics of the drugs in the plasma and lung parenchyma were not measured. The dose used in this study was extrapolated from the literature and our clinical experience. Further studies to determine the best timing for an inhaled milrinone bolus, namely before or after $\mathrm{CPB}$, are ongoing. Finally, a model with young and healthy 8-week-old Landrace swine might not allow confirmation that inhaled milrinone has similar beneficial effects in patients with coronary artery disease and pre-existing pulmonary diseases. Cardioplegic arrest was not used in these experiments but is unlikely to change the effects of CPB on pulmonary endothelial function and gas exchange, although myocardial global ischemia is known to increase inflammation.

\section{Conclusion}

This study comparing the effects of 2 modes of administration of milrinone in swine undergoing CPB showed that inhaled milrinone is safer and is associated with a lower heart rate throughout the operation. It completely prevented the pulmonary endothelial dysfunction and was associated with better oxygen exchange than its intravenous administration in this pulmonary ischemia-reperfusion model. This therapy could be useful in patients at risk for postoperative $\mathrm{PH}$ undergoing cardiac surgery and in other settings of ischemia-reperfusion injuries, such as lung transplantation. Trials in patients undergoing cardiac surgery are ongoing to explore the effects of this mode of administration in the clinical arena. 


\section{References}

1. Kolff WJ, Effler DB, Groves LK, Hughes CR, McCormack LJ. Pulmonary complications of open-heart operations: their pathogenesis and avoidance. Cleve Clin Q. 1958;25:65-83

2. Ng CS, Wan S, Yim AP, Arifi AA. Pulmonary dysfunction after cardiac surgery. Chest. 2002;121:1269-77.

3. Wan S, LeClerc JL, Vincent JL. Inflammatory response to cardiopulmonary bypass: mechanisms involved and possible therapeutic strategies. Chest. 1997;112:676-92.

4. Boyle EM Jr, Pohlman TH, Johnson MC, Verrier ED. Endothelial cell injury in cardiovascular surgery: the systemic inflammatory response. Ann Thorac Surg. 1997;63:277-84.

5. Davila-Roman VG, Waggoner AD, Hopkins WE, Barzilai B. Right ventricular dysfunction in low output syndrome after cardiac operations: assessment by transesophageal echocardiography. Ann Thorac Surg. 1995;60:1081-6.

6. Chen YF, Oparil S. Endothelial dysfunction in the pulmonary vascular bed. Am J Med Sci. 2000;320:223-32.

7. Haraldsson A, Kieler-Jensen N, Ricksen SE. The additive pulmonary vasodilatory effects of inhaled prostacyclin and inhaled milrinone in postcardiac surgery patients with pulmonary hypertension. Anesth Analg. 2001;93:1439-45.
8. Cuffe MS, Califf RM, Adams KF Jr, Benza R, Bourge R, Colucci WS, et al. Short-term intravenous milrinone for acute exacerbation of chronic heart failure: a randomized controlled trial. JAMA. 2002;287:1541-7.

9. Reichert CL, Visser CA, Koolen JJ, vd Brink RB, van Wezel HB, Meyne NG, et al. Transesophageal echocardiography in hypotensive patients after cardiac operations. Comparison with hemodynamic parameters. J Thorac Cardiovasc Surg. 1992;104:321-6.

10. Morita K, Ihnken K, Buckberg GD, Sherman MP, Ignarro LJ. Pulmonary vasoconstriction due to impaired nitric oxide production after cardiopulmonary bypass. Ann Thorac Surg. 1996;61:1775-80.

11. Riedel B. The pathophysiology and management of perioperative pulmonary hypertension with specific emphasis on the period following cardiac surgery. Int Anesthesiol Clin. 1999;37:55-79.

12. Fortier S, DeMaria RG, Lamarche Y, Malo O, Denault A, Desjardins $\mathrm{F}$, et al. Inhaled prostacyclin reduces cardiopulmonary bypass-induced pulmonary endothelial dysfunction via increased cyclic adenosine monophosphate levels. J Thorac Cardiovasc Surg. 2004;128: 109-16.

13. Niwano K, Arai M, Tomaru K, Uchiyama T, Ohyama Y, Kurabayashi M. Transcriptional stimulation of the eNOS gene by the stable prostacyclin analogue beraprost is mediated through cAMP-responsive element in vascular endothelial cells. Circ Res. 2003;93:523-30. 